\title{
Overweight, Obesity, and Late-Life Sarcopenia Among Men With Cardiovascular Disease, Israel
}

\author{
Miri Lutski, $\mathrm{PhD}^{1}$; Galit Weinstein, $\mathrm{PhD}^{2}$; David Tanne, $\mathrm{MD}^{3,4}$; Uri Goldbourt, $\mathrm{PhD}^{5}$
}

Accessible Version: www.cdc.gov/pcd/issues/2020/20_0167.htm

Suggested citation for this article: Lutski M, Weinstein G, Tanne D, Goldbourt U. Overweight, Obesity, and Late-Life Sarcopenia Among Men With Cardiovascular Disease, Israel. Prev Chronic Dis 2020;17:200167. DOI: https://doi.org/10.5888/ pcd17.200167.

\section{PEER REVIEWED}

\section{Summary}

What is known about this topic?

Cardiovascular disease (CVD) might accelerate the development of sarcopenia. However, little is known about the association between obesity and sarcopenia among patients with CVD.

What is added by this report?

We observed a positive association between men who were overweight or obese and had CVD and late-life sarcopenia.

What are the implications for public health?

Given the growth of the population aged 65 or older and the high prevalence of obesity and CVD, it is important to identify people with CVD and potentially preventable risk factors for sarcopenia.

\section{Abstract}

\section{Introduction}

Little is known about the association between obesity and sarcopenia - age-related loss of muscle mass and function - among patients with cardiovascular disease. We investigated the association between overweight, obesity, and sarcopenia among community-dwelling men in Israel with cardiovascular disease.

\section{Methods}

A subset of 337 men (mean age at baseline 56.7 [SD, 6.5]) who previously (1990-1997) participated in the Bezafibrate Infarction Prevention trial underwent a neurovascular evaluation as part of the Bezafibrate Infarction Prevention Neurocognitive Study 15.0 (SD, 3.0) years after baseline and a sarcopenia evaluation 19.9 $(\mathrm{SD}, 1.0)$ years after baseline. We applied a multinomial logistic model to estimate odds ratios and $95 \%$ CIs for 3 categories of sarcopenia: no evidence of sarcopenia (ie, robust), probable sarcopenia, and sarcopenia.

\section{Results}

We found sarcopenia among $54.3 \%$ of participants with obesity (body mass index [BMI, in $\left.\mathrm{kg} / \mathrm{m}^{2}\right] \geq 30.0$ ), $37.0 \%$ of participants who were overweight $(25.0 \leq \mathrm{BMI} \leq 29.9)$, and $24.8 \%$ of participants with normal weight (BMI 18.5 to 24.9). In a comparison of $\mathrm{BMI} \geq 25.0$ and $\mathrm{BMI}<25.0$, adjusting for covariates, the odds ratio of having probable sarcopenia was 3.27 (95\% CI, 1.68-6.36) and having sarcopenia was 5.31 (95\% CI, 2.50-11.27).

\section{Conclusion}

We found a positive association between obesity and late-life sarcopenia and suggest that obesity might be an important modifiable risk factor related to sarcopenia among men with cardiovascular disease.

\section{Introduction}

Sarcopenia, from the Greek "poverty of flesh," is a highly prevalent geriatric syndrome first described by Rosenberg in 1989 as the age-related loss of muscle mass and function (1). Accumulating evidence suggests that sarcopenia is associated with adverse health outcomes such as frailty, falls, disability, admission to nursing homes, and mortality (2). Several underlying mechanisms are linked with the development of sarcopenia, including impaired neuromuscular function, hormonal changes, increased inflammation, changes in body-fat distribution, poor nutritional status, and various chronic conditions, yet not all have been fully elucidated (3). The most studied approach in modifying risk factors for sarcopenia is resistance exercise. Numerous treatments of sarcopenia, including protein supplementation and pharmacological interventions, have limited value (4).

Obesity-mediated factors may aggravate sarcopenia in older people and maximize its effects on physical disability, morbidity, and mortality $(5,6)$. Several studies investigated the association of obesity with sarcopenia (7-10). Findings on the association 
between overweight and sarcopenia are controversial (7). The prevalence of cardiovascular disease (CVD) in middle and old age is increasing, partly as a result of increases in the prevalence of obesity $(11,12)$. Furthermore, CVD might accelerate the development of sarcopenia, and both have been strongly tied to chronic low-grade inflammation, insulin resistance, and obesity (13). However, little is known about the association between obesity and sarcopenia in patients with CVD. The aim of this study was to describe the association between overweight, obesity, and late-life sarcopenia among community-dwelling men aged 64 or older with CVD.

\section{Methods}

Our study sample consisted of a subset of patients from 8 hospitals who resided in the central region of Israel and who previously participated in the Bezafibrate Infarction Prevention (BIP) clinical trial of lipid modification during 1990-1997 $(\mathrm{N}=1,232)$ and then in the BIP Neurocognitive Study during 2004-2013. The study design and procedures of the BIP trial are detailed elsewhere (14). In brief, the BIP study was a placebo-controlled randomized clinical trial investigating the efficacy of a $400-\mathrm{mg}$ daily dose of bezafibrate, a fibric derivative, in secondary prevention among patients with established stable coronary heart disease. To be included in our study, the lipid profile of patients had to fall within these parameters: serum total cholesterol 180 to $250 \mathrm{mg} / \mathrm{dL}$, lowdensity lipoprotein cholesterol $\leq 180 \mathrm{mg} / \mathrm{dL}(\leq 160 \mathrm{mg} / \mathrm{dL}$ for people aged $<50$ ), high-density lipoprotein cholesterol $\leq 45 \mathrm{mg} / \mathrm{dL}$, and triglycerides $\leq 300 \mathrm{mg} / \mathrm{dL}$. Other exclusion criteria were renal failure, defined as a serum creatinine level $\geq 1.5 \mathrm{mg} / \mathrm{dl}$ or nephrotic syndrome; liver failure, defined as serum glutamate pyruvatetransominase $>60 \mathrm{U} / \mathrm{L}$; and stroke, assessed by reviewing records from hospital or emergency department discharge, a primary care physician, or a neurologist (14).

The BIP Neurocognitive Study consisted of 2 follow-up evaluations (15). The first follow-up evaluation (time $1 ; \mathrm{n}=546$ ) was performed during 2004-2009, an average of 15.0 (SD, 3.0) years after recruitment to BIP; it assessed neurovascular and cognitive function. Patients were re-examined during 2011-2013 (time 2; n =351), 19.9 (SD, 1.0) years after recruitment; this examination assessed sarcopenia and re-assessed cognitive function. The mean interval between time 1 and time 2 was 4.8 (SD, 1.3) years. Patients were assessed at a central research center (the Sagol Neuroscience Center, Sheba Medical Center, Ramar Gan, Israel), or if a patient was unable or unwilling to attend the medical center, the assessment occurred at their residence.

The institutional review boards of the Sheba Medical Center Ethics Committee approved the study and informed consent was obtained from all participants.

\section{Baseline measurements (1990-1993)}

Methods for baseline assessment of the BIP study are described elsewhere (16). Height and weight (without shoes) were measured at baseline (1990-1992) using a standard stadiometer and an electronic weighing scale. Body mass index (BMI) was calculated as body weight in kilograms divided by height in meters squared $(\mathrm{kg} /$ $\mathrm{m}^{2}$ ). Weight was categorized as normal (BMI 18.5-24.9, hereinafter referred to as BMI <25.0), overweight (BMI 25.0-29.9), or obese (BMI $\geq 30.0$ ). Blood samples were drawn from each study patient at baseline of the BIP trial. Samples were collected after at least 12 hours of fasting, with the use of standardized equipment and procedures and transferred to a central study laboratory. Insulin resistance was defined as the upper quartile of the homeostatic model assessment of insulin resistance, calculated as fasting insulin $(\mu \mathrm{U} / \mathrm{ml}) \times$ fasting glucose in $\mathrm{mg} / \mathrm{dL} / 405$. C-reactive protein concentrations were measured using standard automated procedures with commercially available kits (Roche Diagnostics). Angina severity at baseline of the BIP study was classified according to the Canadian Cardiovascular Society angina classification, the standard measure for grading the severity of effort-induced angina (17). Participants were categorized into 2 groups: 1) no angina pectoris or angina pectoris class 1 , and 2) angina pectoris class 2 and above. Physical activity was assessed by asking participants, "Do you participate in one or more of the following activities such as walking, swimming, jogging, gymnastics, biking, dancing, tennis, gardening, gym and rehabilitation activities?" Responses were categorized as any physical activity or sedentary physical activity. Data on education, place of birth, smoking, and comorbidity were collected through a questionnaire at baseline.

\section{Sarcopenia evaluation (time 2)}

Sarcopenia was defined by using the European Working Group on Sarcopenia in Older People definitions of low muscle strength, low muscle mass, and low physical performance (2). Probable sarcopenia was defined as low muscle strength or low muscle mass. Sarcopenia was defined as low muscle strength and low muscle mass and/or low physical performance.

Muscle strength was defined as isometric dominant handgrip strength, which was assessed by using a Jamar hydraulic hand dynamometer (Sammons Preston). Muscle strength was categorized as low if $\leq 29 \mathrm{~kg}$ (for patients with BMI $\leq 24.0$ ), $\leq 30 \mathrm{~kg}$ (for patients with BMI 24.1-28.0), and $\leq 32 \mathrm{~kg}$ (for patients with BMI $>28.0$ ). The test was carried out twice and the higher score was used.

Muscle mass was assessed by bioelectric impedance analysis using a Tanita BC-545 8-contact electrode body composition analyzer. To calculate body composition, the computer software in the

The opinions expressed by authors contributing to this journal do not necessarily reflect the opinions of the U.S. Department of Health and Human Services, the Public Health Service, the Centers for Disease Control and Prevention, or the authors' affiliated institutions. 
bioelectric impedance analysis system uses the measured impedance, the programmed person's sex and height, and the measured weight. The device measures fat-free mass in kilograms and the percentage of total body fat according to the equation provided by the device's software. Skeletal muscle mass was calculated by using the following equation: skeletal muscle mass $=0.566 \times$ fat-free mass. Skeletal muscle mass index, adjusted for weight, was calculated as (skeletal muscle mass $\times 100) /$ weight $(18)$. Low skeletal muscle mass index was defined as $37.4 \%$ or lower (19).

We used gait speed to determine physical performance. Gait speed was measured by gait time in seconds using a 5-meter timed walk test. Usual gait speed of less than 1 meter per second signifies a high risk of health-related outcomes in well-functioning older people (20). The test instructions were as follows: "On the word 'go' start walking at your regular pace to the line on the floor." We used height-adjusted time as the cutoff. Low physical performance was denoted as $\geq 6$ seconds (for height $\leq 173 \mathrm{~cm}[68$ inches]) and $\geq 5$ seconds (for height $>173 \mathrm{~cm}$ ) $(21)$.

\section{Additional assessments}

In both evaluations (time 1 and time 2), data on comorbidities and hospitalizations, medication use, smoking status, physical activity, and anthropometric measurements were collected systematically. In addition, systolic blood pressure, diastolic blood pressure, and weight and height were measured.

Incident stroke during follow-up was assessed by reviewing records from hospital or emergency department discharge, primary care physicians, or neurologists. We used a score of 5 or more on the 15-item version of the Geriatric Depression Scale (22) to indicate clinically significant depressive symptoms. Patients completed the NeuroTrax computerized cognitive test (NeuroTrax Corporation). A description of this test is available elsewhere (23). All NeuroTrax scores are normalized according to age- and education-specific normative data and scaled to an IQ-style scale with a mean of 100 and SD of 15 . Dementia and incident stroke during follow-up were determined by an adjudication committee composed of 3 investigators, 2 of whom were experienced board certified neurologists. A diagnosis of dementia was based on a cognitive evaluation, a clinical interview, and data collected and was in accordance with criteria of the Diagnostic and Statistical Manual of Mental Disorders, 4th Edition (24). An occurrence of stroke was determined on the basis of World Health Organization criteria (25).

Cerebrovascular reactivity, a marker of cerebral microvascular function, was evaluated by transcranial Doppler (Trans-Link 9900 [Rimed]), which measures the breath-holding index (26). Participants were categorized into normal $(\geq 0.69)$ or impaired $(<0.69)$ cerebrovascular reactivity on the basis of the mean breath-holding index of both middle cerebral arteries according to previously established standard parameters (27). Carotid intima-media thickness (cIMT), a measure of cerebral large-vessel atherosclerosis, and plaque presence were measured at the far wall of both common carotid arteries using high-resolution B-mode ultrasound. Participants were classified into the following 2 categories according to previously established standard parameters (28): cIMT $\geq 0.93 \mathrm{~mm}$ (elevated) and/or bilateral carotid plaques or cIMT $<0.93$ (normal) and without bilateral carotid plaques.

\section{Statistical analysis}

We summarized data on the clinical characteristics of patients as percentage and mean (SD), unless the distribution was strongly skewed, in which case we summarized data as median and interquartile range (IQR). We compared variables between BMI and sarcopenia groups by using analysis of variance or the Kruskal-Wallis test for continuous variables and the $\chi^{2}$ test for categorical variables. We used multinomial logistic regression to estimate odds ratios (ORs) and 95\% CIs for sarcopenia. The categories of the outcome variable were sarcopenia, probable sarcopenia, and robust (ie, no evidence of sarcopenia); we used robust as the reference category in comparisons. We first adjusted for age, education, and birthplace, then additionally for systolic blood pressure, physical activity, diabetes, insulin resistance, Creactive protein, high-density lipoprotein cholesterol, and triglycerides. Subsequently, we further adjusted for depressive symptoms (Geriatric Depression Scale score $\geq 5$ vs $<5$ ), global cognitive score, and Doppler ultrasound indices of cerebrovascular disease at time 1 .

Because of loss to follow-up of eligible patients who had either died or refused participation, we estimated the probability of every person to reach the sarcopenia assessment and calculated the inverse probability weights (29). We compared the results of weighted analysis with nonweighted analysis. Data were analyzed using SPSS version 21 (IBM Corporation).

\section{Results}

Of the 1,232 patients eligible for the evaluation at time 1, 214 had died; 259 refused to participate; 102 could not be contacted; 45 were unable to participate because of dementia, language incompatibility, vision or hearing defects, or physical disability; and 54 were excluded because of missing data. Twelve women were not included because of the small sample. This process of exclusion resulted in 546 men available for evaluation at time 1 (Figure). A total of 351 patients were re-assessed at time 2; of these, 337 had BMI measurements and a sarcopenia evaluation. The attrition from time 1 and time 2 was mainly a result of interim death $(\mathrm{n}=$

The opinions expressed by authors contributing to this journal do not necessarily reflect the opinions of the U.S. Department of Health and Human Services, the Public Health Service, the Centers for Disease Control and Prevention, or the authors' affiliated institutions. 
$114)$; in addition, 58 refused to participate, 4 could not be contacted, and 19 were unable to participate. The mean (SD) age of the study sample was $56.7(6.5)$ at baseline, $71.8(6.5)$ at time 1, and $77.1(6.4)$ at time 2.

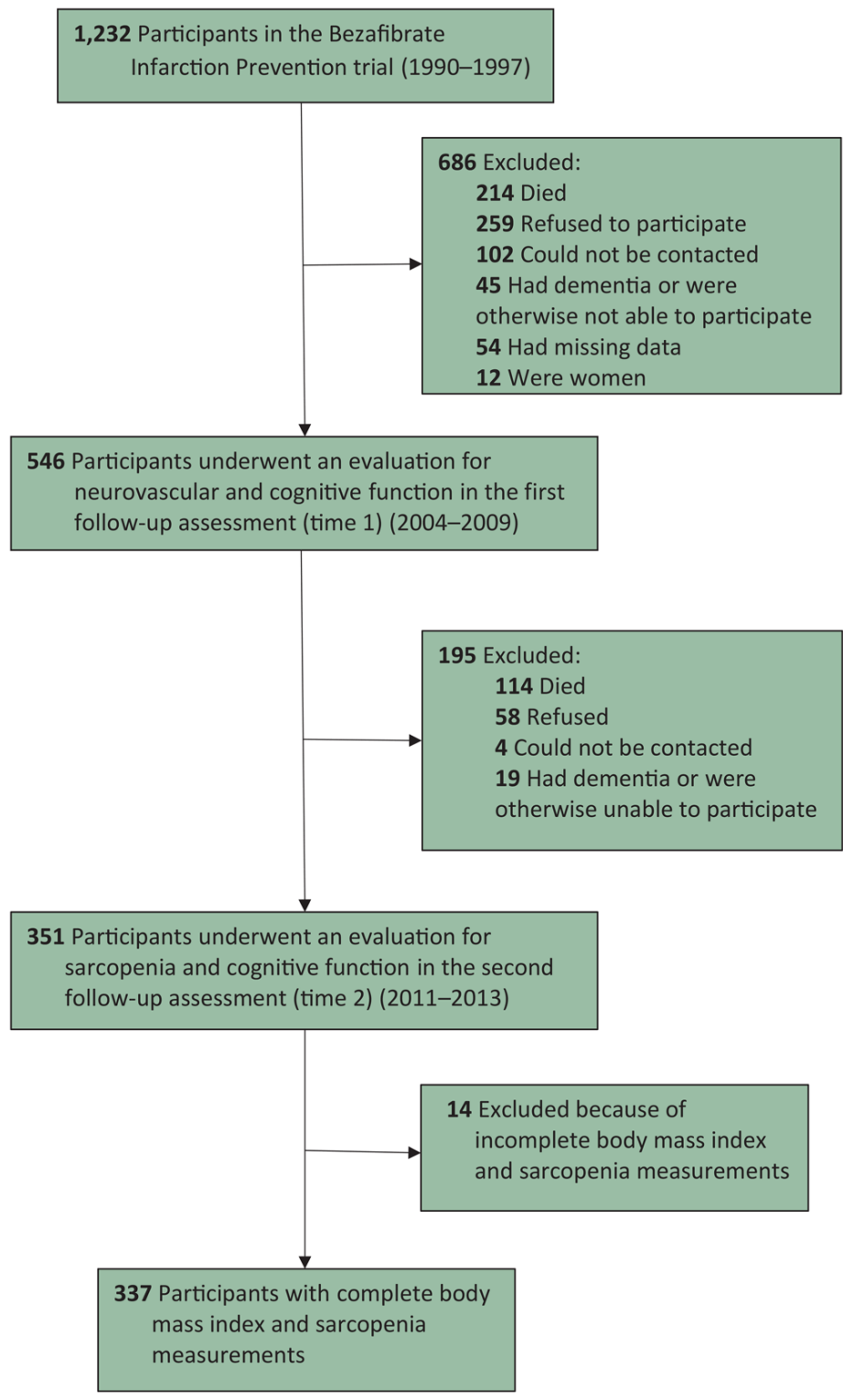

Figure. Study flowchart. Participants from 8 hospitals in central Israel were initially recruited for the Bezafibrate Infarction Prevention (BIP) clinical trial of lipid modification during 1990-1997 and were also in the BIP Neurocognitive Study. The BIP Neurocognitive Study consisted of 2 follow-up evaluations: time 1 (2004-2009) and time 2 (2011-2013).

Of the 337 patients, 109 (32.3\%) were classified as robust, 112 $(33.2 \%)$ as probable sarcopenia, and $116(34.4 \%)$ as sarcopenia (Table 1). Sarcopenia was significantly related to older age, less education, more severe angina pectoris (angina pectoris class $\geq 2$ ), and a higher systolic blood pressure at baseline. In addition, participants with sarcopenia had poorer global cognitive function, more cerebrovascular diseases, and a higher level of depression at time 1 compared with their counterparts.

In general, patients with obesity, compared with patients without obesity, more often had diabetes and insulin resistance, a blood glucose level $\geq 100 \mathrm{mg} / \mathrm{dL}$, and higher levels of C-reactive protein, and they were less physically active at baseline. We found sarcopenia among $54.3 \%$ of patients with obesity, $37.0 \%$ of patients who were overweight, and $24.8 \%$ of patients with normal weight ( $P$ for trend $<.001)$.

Adjusting for age, education, birthplace, systolic blood pressure, physical activity, insulin resistance, C-reactive protein, highdensity lipid cholesterol, and triglycerides, the estimated adjusted OR $(95 \% \mathrm{CI})$ for probable sarcopenia for patients with BMI $\geq 25.0$, compared with patients with BMI $<25.0$, was 2.88 (1.54-5.36) and for sarcopenia was 5.04 (2.51-10.15). Additional adjustment for global cognitive score, Doppler ultrasound indices of cerebrovascular disease, and depressive symptoms did not materially alter the results (Table 2). An increment of 1.0 BMI unit was associated with an adjusted OR (95\% CI) of 1.33 (1.16-1.53) for probable sarcopenia and of 1.38 (1.19-1.59) for sarcopenia (Table 3$)$. The adjusted OR (95\% CI) for probable sarcopenia for patients with obesity was $6.51(1.56-27.13)$ and for patients who were overweight (but not obese) was 3.03 (1.54-5.98). The adjusted OR $(95 \% \mathrm{CI})$ for sarcopenia for patients with obesity was 13.49 (3.12-58.20) and for patients who were overweight was 4.65 (2.16-10.04).

\section{Discussion}

In this cross-sectional study of men with CVD, being overweight and obese in their fifties was related to late-life sarcopenia. The observed relationship was independent of traditional cardiovascular risk factors and global cognitive score and did not change after exclusion of participants with a history of stroke or dementia at the sarcopenia assessment. To the best of our knowledge, our study is the first to estimate the association between obesity and overweight and late-life sarcopenia among men with CVD. In our sample, the prevalence of probable sarcopenia $(33.2 \%)$ and sarcopenia (34.4\%) was high compared with the prevalence in the general population $(5 \%-13 \%)$ measured by using the criteria of the European Working Group on Sarcopenia in Older People (2). A retrospective study of Japanese men with CVD and an average age of $72(\mathrm{SD}, 12)$ found sarcopenia in $29.5 \%$ of the study population (30). The prevalence of sarcopenia is higher among people with CVD than among the general population, but comparisons of

The opinions expressed by authors contributing to this journal do not necessarily reflect the opinions of the U.S. Department of Health and Human Services, the Public Health Service, the Centers for Disease Control and Prevention, or the authors' affiliated institutions. 
sarcopenia rates between studies are difficult because of differences in study population, age ranges, sarcopenia definition, and the use of various thresholds for defining low muscle mass and low muscle performance $(10,31)$.

Some researchers have suggested that being overweight in midlife may be associated with lower, rather than higher, sarcopenia rates (32). In a 4-year follow-up study among community-dwelling Chinese men and women aged 72.5 (SD, 5.2) on average found that higher BMI was inversely associated with the development of sarcopenia (7). On the other hand, in the Collaborative Research on Ageing in Europe survey conducted among people aged 65 or older, higher percentage body fat and lower levels of physical activity were associated with low muscle mass and sarcopenia in almost all the countries studied (10).

Obesity is associated with increased risk of glucose intolerance, chronic inflammation, hypertension, dyslipidemia, and CVD. Sarcopenia shares many pathological mechanisms with obesity, including insulin resistance and low-grade chronic inflammation $(3,5)$. Aging induces changes in body composition, such as increases in visceral fat and decreases in skeletal muscle mass (10). Fat mass induces inflammation, which may contribute to the development of sarcopenia (33). A vicious circle between muscle loss and fat gain may lead to more sarcopenia and then to further weight gain, inflammation, and impaired glucose tolerance (5). However, these changes in body composition, such as increases in fat and decreases in muscle mass, are potentially reversible by modifying lifestyle behaviors (7,33). Resistance exercise and nutrition support, which increases muscle mass and muscle strength, are key lifestyle strategies that can prevent sarcopenia or reverse it (34).

Strengths of this study include a unique data set of extensively studied men with CVD who underwent comprehensive evaluations. Skeletal muscle mass was assessed by bioimpedance method, which is considered simple and inexpensive and has a high correlation with magnetic resonance imaging and dual-energy $\mathrm{x}$ ray absorptiometry (19). However, some important issues need to be considered. First, we could not evaluate the causal effect of obesity on sarcopenia because we did not assess sarcopenia at baseline. Second, the generalizability of the study is limited to men with CVD who had the specific clinical characteristics required for eligibility in the BIP study. Finally, despite an acceptable response rate of $82 \%$ among survivors, a substantial proportion of patients did not participate in the late-life assessment because of the long period between baseline and the late-life evaluations.

In summary, we observed a positive association between overweight and obese men with CVD and late-life sarcopenia. Given the growth of the population aged 65 or older and the high prevalence of obesity and CVD $(11,12)$, it is important to identify people with CVD and potentially preventable risk factors for sarcopenia. However, these findings need to be confirmed in larger studies.

\section{Acknowledgments}

The authors declared no potential conflicts of interest with respect to the research, authorship, and/or publication of this article. The authors received no financial support for the research, authorship, and/or publication of this article. No copyrighted materials were used in this research.

\section{Author Information}

Corresponding Author: Miri Lutski, PhD, The Israel Center for Disease Control, Ministry of Health, Gertner Building, Sheba Medical Center, Tel Hashomer 52621 Israel. Telephone: +972 37651501. Email: Miri77@gmail.com.

Author Affiliations: ${ }^{1}$ The Israel Center for Disease Control, Israel Ministry of Health, Tel Hashomer, Israel. ${ }^{2}$ School of Public Health, Faculty of Social Welfare and Health Sciences, University of Haifa, Haifa, Israel. ${ }^{3}$ Department of Neurology, Sackler Faculty of Medicine, Tel Aviv University, Tel Aviv, Israel. ${ }^{4}$ Stroke and Cognition Institute, Rambam Health Care Campus, Haifa, Israel. ${ }^{5}$ Department of Epidemiology and Preventive Medicine, School of Public Health, Sackler Faculty of Medicine, Tel Aviv University, Tel Aviv, Israel.

\section{References}

1. Rosenberg IH. Sarcopenia: origins and clinical relevance. J Nutr 1997;127(5Suppl):990S-1S.

2. Cruz-Jentoft AJ, Bahat G, Bauer J, Boirie Y, Bruyère O, Cederholm T, et al.; Writing Group for the European Working Group on Sarcopenia in Older People 2 (EWGSOP2), and the Extended Group for EWGSOP2. Sarcopenia: revised European consensus on definition and diagnosis. Age Ageing 2019; 48(1):16-31.

3. Walrand S, Guillet C, Salles J, Cano N, Boirie Y. Physiopathological mechanism of sarcopenia. Clin Geriatr Med 2011;27(3):365-85.

4. Kilsby AJ, Sayer AA, Witham MD. Selecting potential pharmacological interventions in sarcopenia. Drugs Aging 2017;34(4):233-40.

5. Zamboni M, Mazzali G, Fantin F, Rossi A, Di Francesco V. Sarcopenic obesity: a new category of obesity in the elderly. Nutr Metab Cardiovasc Dis 2008;18(5):388-95.

The opinions expressed by authors contributing to this journal do not necessarily reflect the opinions of the U.S. Department of Health and Human Services, the Public Health Service, the Centers for Disease Control and Prevention, or the authors' affiliated institutions. 
6. Kalinkovich A, Livshits G. Sarcopenic obesity or obese sarcopenia: a cross talk between age-associated adipose tissue and skeletal muscle inflammation as a main mechanism of the pathogenesis. Ageing Res Rev 2017;35:200-21.

7. Yu R, Wong M, Leung J, Lee J, Auyeung TW, Woo J. Incidence, reversibility, risk factors and the protective effect of high body mass index against sarcopenia in communitydwelling older Chinese adults. Geriatr Gerontol Int 2014; 14(Suppl 1):15-28.

8. Gabat JAL, Faltado AL Jr, Sedurante MB, Tee ML. Association of obesity and sarcopenia among adult Filipinos. Osteoporos Sarcopenia 2018;4(3):109-13.

9. Han P, Kang L, Guo Q, Wang J, Zhang W, Shen S, et al. Prevalence and factors associated with sarcopenia in suburbdwelling older Chinese using the Asian Working Group for Sarcopenia definition. J Gerontol A Biol Sci Med Sci 2016; 71(4):529-35.

10. Tyrovolas S, Koyanagi A, Olaya B, Ayuso-Mateos JL, Miret M, Chatterji S, et al. Factors associated with skeletal muscle mass, sarcopenia, and sarcopenic obesity in older adults: a multi-continent study. J Cachexia Sarcopenia Muscle 2016; 7(3):312-21.

11. Lechleitner M. Obesity and the metabolic syndrome in the elderly — a mini-review. Gerontology 2008;54(5):253-9.

12. Mendis S, Banerjee A. Cardiovascular disease: equity and social determinants. In: Blas E, Kurup AS, editors. Equity, social determinants and public health programmes. Geneva (CH): World Health Organization; 2010.

13. Wannamethee SG, Atkins JL. Muscle loss and obesity: the health implications of sarcopenia and sarcopenic obesity. Proc Nutr Soc 2015;74(4):405-12.

14. Goldbourt U, Behar S, Reicher-Reiss H, Agmon J, Kaplinsky E, Graff E, et al. Rationale and design of a secondary prevention trial of increasing serum high-density lipoprotein cholesterol and reducing triglycerides in patients with clinically manifest atherosclerotic heart disease (the Bezafibrate Infarction Prevention Trial). Am J Cardiol 1993; 71(11):909-15.

15. Lutski M, Tanne D, Goldbourt U. Tall stature in coronary heart disease patients is associated with decreased risk of frailty in late life. Geriatr Gerontol Int 2017;17(9):1270-7.

16. Goldbourt U, Brunner D, Behar S, Reicher-Reiss H. Baseline characteristics of patients participating in the Bezafibrate Infarction Prevention (BIP) Study. Eur Heart J 1998;19(Suppl H):H42-7.

17. Campeau L. Letter: Grading of angina pectoris. Circulation 1976;54(3):522-3.

18. Janssen I, Heymsfield SB, Baumgartner RN, Ross R. Estimation of skeletal muscle mass by bioelectrical impedance analysis. J Appl Physiol (1985) 2000;89(2):465-71.
19. Bahat G, Tufan A, Kilic C, Öztürk S, Akpinar TS, Kose M, et al. Cut-off points for weight and body mass index adjusted bioimpedance analysis measurements of muscle mass. Aging Clin Exp Res 2019;31(7):935-42.

20. Cesari M, Kritchevsky SB, Penninx BW, Nicklas BJ, Simonsick EM, Newman AB, et al. Prognostic value of usual gait speed in well-functioning older people - results from the Health, Aging and Body Composition Study. J Am Geriatr Soc 2005;53(10):1675-80.

21. Fried LP, Tangen CM, Walston J, Newman AB, Hirsch C, Gottdiener J, et al.; Cardiovascular Health Study Collaborative Research Group. Frailty in older adults: evidence for a phenotype. J Gerontol A Biol Sci Med Sci 2001; 56(3):M146-56.

22. Almeida OP, Almeida SA. Short versions of the geriatric depression scale: a study of their validity for the diagnosis of a major depressive episode according to ICD-10 and DSM-IV. Int J Geriatr Psychiatry 1999;14(10):858-65.

23. Dwolatzky T, Whitehead V, Doniger GM, Simon ES, Schweiger A, Jaffe D, et al. Validity of a novel computerized cognitive battery for mild cognitive impairment. BMC Geriatr 2003;3(1):4.

24. American Psychiatric Association. Diagnostic and statistical manual of mental disorders. 4th ed. Washington (DC): American Psychiatric Association; 1994.

25. Hatano S. Experience from a multicentre stroke register: a preliminary report. Bull World Health Organ 1976; 54(5):541-53.

26. Müller M, Voges M, Piepgras U, Schimrigk K. Assessment of cerebral vasomotor reactivity by transcranial Doppler ultrasound and breath-holding. A comparison with acetazolamide as vasodilatory stimulus. Stroke 1995; 26(1):96-100.

27. Silvestrini M, Vernieri F, Pasqualetti P, Matteis M, Passarelli F, Troisi E, et al. Impaired cerebral vasoreactivity and risk of stroke in patients with asymptomatic carotid artery stenosis. JAMA 2000;283(16):2122-7.

28. Lorenz MW, Polak JF, Kavousi M, Mathiesen EB, Völzke H, Tuomainen TP, et al.; PROG-IMT Study Group. Carotid intima-media thickness progression to predict cardiovascular events in the general population (the PROG-IMT collaborative project): a meta-analysis of individual participant data. Lancet 2012;379(9831):2053-62.

29. Robins JM, Hernán MA, Brumback B. Marginal structural models and causal inference in epidemiology. Epidemiology 2000;11(5):550-60.

30. Harada H, Kai H, Shibata R, Niiyama H, Nishiyama Y, Murohara $\mathrm{T}$, et al. New diagnostic index for sarcopenia in patients with cardiovascular diseases. PLoS One 2017; 12(5):e0178123.

The opinions expressed by authors contributing to this journal do not necessarily reflect the opinions of the U.S. Department of Health and Human Services, the Public Health Service, the Centers for Disease Control and Prevention, or the authors' affiliated institutions.

6 Centers for Disease Control and Prevention • www.cdc.gov/pcd/issues/2020/20_0167.htm 
31. Cawthon PM, Travison TG, Manini TM, Patel S, Pencina KM, Fielding RA, et al. Establishing the link between lean mass and grip strength cut-points with mobility disability and other health outcomes: proceedings of the Sarcopenia Definition and Outcomes Consortium Conference. J Gerontol A Biol Sci Med Sci 2020;75(7):1317-23. .

32. Kirchengast S, Huber J. Mild overweight reduces the risk of sarcopenia in healthy women. Open Womens Health J 2010; $4(1): 62-8$.

33. Gregor MF, Hotamisligil GS. Inflammatory mechanisms in obesity. Annu Rev Immunol 2011;29(1):415-45.

34. Fiatarone MA, O'Neill EF, Ryan ND, Clements KM, Solares GR, Nelson ME, et al. Exercise training and nutritional supplementation for physical frailty in very elderly people. N Engl J Med 1994;330(25):1769-75.

The opinions expressed by authors contributing to this journal do not necessarily reflect the opinions of the U.S. Department of Health and Human Services, the Public Health Service, the Centers for Disease Control and Prevention, or the authors' affiliated institutions. 


\section{Tables}

Table 1. Baseline Characteristics of Participants in the Bezafibrate Infarction Prevention Neurocognitive Study, by Sarcopenia Status at Time 2, Israel, 2011-2013

\begin{tabular}{|c|c|c|c|c|}
\hline \multirow[b]{2}{*}{ Characteristic } & \multicolumn{4}{|c|}{ Sarcopenia Status $(\mathrm{N}=337)$} \\
\hline & Robust $^{a}(n=109)$ & $\begin{array}{l}\text { Probable Sarcopenia }{ }^{b} \\
(n=112)\end{array}$ & Sarcopenia $^{c}(n=116)$ & $P$ Value $^{\mathrm{d}}$ \\
\hline \multicolumn{5}{|c|}{ Baseline (1990-1993) } \\
\hline Age, mean (SD), y & $54.0(5.7)$ & $55.6(6.4)$ & $59.9(5.8)$ & $<.001$ \\
\hline$<12$ Years of education, $n(\%)$ & $25(22.9)$ & $37(33.0)$ & $43(37.1)$ & .02 \\
\hline \multicolumn{5}{|l|}{ Birthplace, n (\%) } \\
\hline Middle East & $32(29.4)$ & $33(29.5)$ & 39 (33.6) & $.02^{\mathrm{e}}$ \\
\hline Europe & $47(43.1)$ & $46(41.1)$ & $55(47.4)$ & \\
\hline Israel & $30(27.5)$ & $33(29.5)$ & $22(19.0)$ & \\
\hline \multicolumn{5}{|l|}{ Smoking, n (\%) } \\
\hline Currently & $5(4.6)$ & $10(8.9)$ & $9(7.8)$ & \multirow[t]{3}{*}{.61} \\
\hline Former & $75(68.8)$ & $67(59.8)$ & $73(62.9)$ & \\
\hline Never & $29(26.6)$ & $35(31.2)$ & $34(29.3)$ & \\
\hline Previous myocardial infarction, $\mathrm{n}(\%)$ & $87(79.8)$ & $92(82.1)$ & $87(75.0)$ & .40 \\
\hline Diabetes, n (\%) & $10(9.2)$ & $9(8.0)$ & $13(11.2)$ & .71 \\
\hline Chronic kidney disease, ${ }^{f} n(\%)$ & $26(23.9)$ & $24(21.6)$ & $39(33.6)$ & .09 \\
\hline Insulin resistance, ${ }^{\mathrm{g}} \mathrm{n}(\%)$ & $25(23.6)$ & $32(30.5)$ & $23(20.9)$ & .25 \\
\hline \multicolumn{5}{|l|}{ Angina class, ${ }^{\mathrm{h}} \mathrm{n}(\%)$} \\
\hline$\geq 2$ & $14(12.8)$ & $14(12.5)$ & $18(15.5)$ & \multirow[t]{2}{*}{$.03^{\mathrm{e}}$} \\
\hline$<2$ & $95(87.2)$ & $98(87.5)$ & $98(84.5)$ & \\
\hline Previous hypertension, $\mathrm{n}(\%)$ & $22(20.2)$ & $31(27.7)$ & $41(35.3)$ & .04 \\
\hline Any physical activity, $\mathrm{n}(\%)$ & $83(78.3)$ & $80(72.1)$ & $82(71.3)$ & .44 \\
\hline Blood glucose $\geq 100 \mathrm{mg} / \mathrm{dL}, \mathrm{n}(\%)$ & $34(31.2)$ & $33(29.7)$ & $51(44.0)$ & .04 \\
\hline \multicolumn{5}{|l|}{ Body mass index, n (\%) } \\
\hline 18.5 to $<25.0 \mathrm{~kg} / \mathrm{m}^{2}$ & $57(52.3)$ & $34(30.4)$ & $30(25.9)$ & \multirow[t]{3}{*}{$<.001$} \\
\hline $25.0-29.9 \mathrm{~kg} / \mathrm{m}^{2}$ & $48(44.0)$ & $66(58.9)$ & $67(57.8)$ & \\
\hline$\geq 30.0 \mathrm{~kg} / \mathrm{m}^{2}$ & $4(3.7)$ & $12(10.7)$ & $19(16.4)$ & \\
\hline Height, mean (SD), m & $1.7(0.6)$ & $1.7(0.7)$ & $1.7(0.6)$ & $<.001$ \\
\hline
\end{tabular}

Abbreviation: IQR, interquartile range.

${ }^{\text {a }}$ No evidence of sarcopenia.

${ }^{\mathrm{b}}$ Defined as low muscle strength or low muscle mass according to European Working Group on Sarcopenia in Older People (2).

${ }^{c}$ Defined as low muscle strength and low muscle mass and/or low physical performance according to European Working Group on Sarcopenia in Older People (2).

${ }^{d} P$ value determined by analysis of variance or Kruskal-Wallis test for continuous variables and $x^{2}$ test for categorical variables, unless otherwise indicated; $P<$

.05 considered significant.

${ }^{\mathrm{e}} P$ for trend determined by $\mathrm{x}^{2}$ test; $P<.05$ considered significant.

${ }^{f}$ Defined as estimated glomerular filtration rate $<60 \mathrm{~mL} / \mathrm{min} / \mathrm{m}^{2}$.

${ }^{g}$ Defined as homeostatic model assessment of insulin resistance in the top quartile $(\geq 1.60)$.

${ }^{\mathrm{h}}$ Classfication according to Canadian Cardiovascular Society angina classification (17); the larger the value, the greater the severity.

i Median (IQR) presented because of nonnormal distribution of data.

${ }^{j}$ Global cognitive score scaled to an IQ-style scale with mean of 100 and SD of 15. Patients completed the NeuroTrax computerized cognitive test (NeuroTrax Corporation). A description of this test is available elsewhere (23).

${ }^{k}$ Geriatric Depression Scale (22) from 0 to 15 ; score of $\geq 5$ indicates clinically significant depressive symptoms.

(continued on next page)

The opinions expressed by authors contributing to this journal do not necessarily reflect the opinions of the U.S. Department of Health and Human Services, the Public Health Service, the Centers for Disease Control and Prevention, or the authors' affiliated institutions. 
(continued)

Table 1. Baseline Characteristics of Participants in the Bezafibrate Infarction Prevention Neurocognitive Study, by Sarcopenia Status at Time 2, Israel, 2011-2013

\begin{tabular}{|c|c|c|c|c|}
\hline \multirow[b]{2}{*}{ Characteristic } & \multicolumn{4}{|c|}{ Sarcopenia Status $(\mathrm{N}=337$ ) } \\
\hline & Robust $^{a}(n=109)$ & $\begin{array}{l}\text { Probable Sarcopenia }{ }^{b} \\
\quad(n=112)\end{array}$ & Sarcopenia $^{c}(n=116)$ & $P$ Value $^{\mathrm{d}}$ \\
\hline \multicolumn{5}{|l|}{ Blood pressure, mean (SD), mm Hg } \\
\hline Systolic & $126(15)$ & $129(15)$ & $133(17)$ & $<.001$ \\
\hline Diastolic & $80(9)$ & $80(8)$ & $81(8)$ & .66 \\
\hline \multicolumn{5}{|l|}{ Cholesterol, mean (SD), mg/dL } \\
\hline Total & $215(16)$ & $214(19)$ & $213(19)$ & .58 \\
\hline Low-density lipoprotein & $150(16)$ & $149(17)$ & $150(18)$ & .91 \\
\hline High-density lipoprotein & $34(5)$ & $35(5)$ & $35(5)$ & .35 \\
\hline Triglycerides, median (IQR), mg/dL ${ }^{i}$ & $141(121-184)$ & $135(108-190)$ & $133(98-174)$ & .08 \\
\hline C-reactive protein, median (IQR), mg/dL ${ }^{i}$ & $2.2(1.1-4.5)$ & $2.1(1.3-4.0)$ & $2.4(1.5-4.9)$ & .23 \\
\hline \multicolumn{5}{|c|}{ Time 1 (2004-2009) } \\
\hline Age, mean (SD), y & $68.8(5.4)$ & $70.7(6.4)$ & $75.3(5.8)$ & $<.001$ \\
\hline Common carotid intima-media thickness, mean (SD), mm & $0.93(0.2)$ & $0.97(0.2)$ & $0.10(0.2)$ & .04 \\
\hline Impaired cerebrovascular reactivity, n (\%) & $41(39.4)$ & $33(32.7)$ & $56(51.4)$ & .02 \\
\hline Bilateral carotid plaque, $\mathrm{n}(\%)$ & $49(45.4)$ & $51(47.2)$ & $69(61.6)$ & .03 \\
\hline Global cognitive score, ${ }^{\mathrm{j}}$ mean (SD) & $98.8(8.9)$ & $96.8(10.0)$ & $95.2(8.9)$ & .02 \\
\hline Geriatric Depression Scale, ${ }^{\mathrm{k}}$ score $\geq 5, \mathrm{n}(\%)$ & $9(8.3)$ & $17(15.2)$ & $26(22.8)$ & $<.001$ \\
\hline \multicolumn{5}{|c|}{ Time 2 (2011-2013) } \\
\hline Age, mean (SD), y & $74.2(5.5)$ & $76.0(6.4)$ & $80.4(5.7)$ & $<.001$ \\
\hline
\end{tabular}

Abbreviation: IQR, interquartile range.

${ }^{\mathrm{a}}$ No evidence of sarcopenia.

${ }^{\mathrm{b}}$ Defined as low muscle strength or low muscle mass according to European Working Group on Sarcopenia in Older People (2).

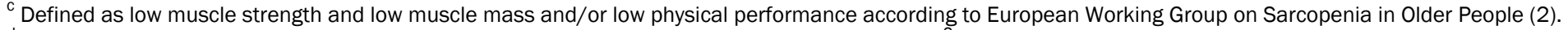

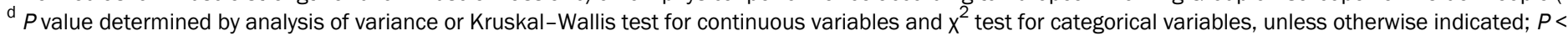

.05 considered significant.

e $P$ for trend determined by $x^{2}$ test; $P<.05$ considered significant.

${ }^{f}$ Defined as estimated glomerular filtration rate $<60 \mathrm{~mL} / \mathrm{min} / \mathrm{m}^{2}$.

${ }^{g}$ Defined as homeostatic model assessment of insulin resistance in the top quartile $(\geq 1.60)$.

${ }^{\mathrm{h}}$ Classfication according to Canadian Cardiovascular Society angina classification (17); the larger the value, the greater the severity.

${ }^{\mathrm{i}}$ Median (IQR) presented because of nonnormal distribution of data.

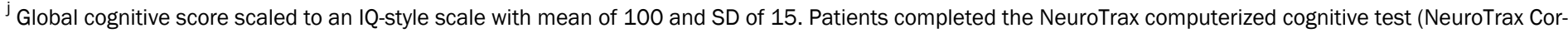
poration). A description of this test is available elsewhere (23).

${ }^{\mathrm{k}}$ Geriatric Depression Scale (22) from 0 to 15; score of $\geq 5$ indicates clinically significant depressive symptoms. 
Table 2. Multinomial Logistic Regression for Association Between BMI Groups ( $\geq 25.0$ vs $<25.0$ ) at Baseline (1990-1993) and Sarcopenia Status at Time 2 (2011-2013) Among a Sample of Men ( $n=337)$ Participating in Bezafibrate Infarction Prevention Neurocognitive Study, Israel ${ }^{a}$

\begin{tabular}{|c|c|c|c|c|}
\hline \multirow[b]{2}{*}{ Model } & \multicolumn{2}{|l|}{ Probable Sarcopenia } & \multicolumn{2}{|l|}{ Sarcopenia } \\
\hline & OR $(95 \% \mathrm{Cl})$ & $P$ Value & OR $(95 \% \mathrm{Cl})$ & $P$ Value \\
\hline \multicolumn{5}{|l|}{ Model $1^{b}$} \\
\hline $\mathrm{BMI} \geq 25$ & $2.95(1.64-5.29)$ & \multirow[t]{2}{*}{$<.001$} & $4.94(2.57-9.48)$ & \multirow[t]{2}{*}{$<.001$} \\
\hline $\mathrm{BMI}<25$ & 1 [Reference] & & 1 [Reference] & \\
\hline \multicolumn{5}{|l|}{ Model $2^{c}$} \\
\hline $\mathrm{BMI} \geq 25$ & $2.88(1.54-5.36)$ & \multirow[t]{2}{*}{.001} & $5.04(2.51-10.15)$ & \multirow[t]{2}{*}{$<.001$} \\
\hline $\mathrm{BMI}<25$ & 1 [Reference] & & 1 [Reference] & \\
\hline \multicolumn{5}{|l|}{ Model $3^{d}$} \\
\hline $\mathrm{BMI} \geq 25$ & $3.27(1.68-6.36)$ & \multirow[t]{2}{*}{$<.001$} & $5.31(2.50-11.27)$ & \multirow[t]{2}{*}{$<.001$} \\
\hline $\mathrm{BMI}<25$ & 1 [Reference] & & 1 [Reference] & \\
\hline \multicolumn{5}{|l|}{ Model $4^{e}$} \\
\hline $\mathrm{BMI} \geq 25$ & $2.72(1.81-4.09)$ & \multirow[t]{2}{*}{$<.001$} & $4.52(2.89-7.05)$ & \multirow[t]{2}{*}{$<.001$} \\
\hline $\mathrm{BMI}<25$ & 1 [Reference] & & 1 [Reference] & \\
\hline \multicolumn{5}{|l|}{ Model $5^{f}$} \\
\hline $\mathrm{BMI} \geq 25$ & $3.76(1.84-7.68)$ & \multirow[t]{2}{*}{$<.001$} & $7.78(3.24-18.69)$ & \multirow[t]{2}{*}{$<.001$} \\
\hline $\mathrm{BMI}<25$ & 1 [Reference] & & 1 [Reference] & \\
\hline
\end{tabular}

Abbreviations: BMI, body mass index; OR, odds ratio.

${ }^{a}$ In all comparisons, reference outcome value is robust, defined as no evidence of sarcopenia. The category BMI <25 excludes underweight (BMI <18.5).

${ }^{\mathrm{b}}$ Model 1 = age, education ( $\geq 12 \mathrm{y}$ vs $<12 \mathrm{y}$ ), and birthplace (Europe, Middle East vs Israel).

${ }^{c}$ Model 2 = Model $1+$ systolic blood pressure (continuous), physical activity, diabetes, insulin resistance (top quartile vs others), C-reactive protein, high-density lipoprotein cholesterol, and triglycerides (continuous).

${ }^{d}$ Model 3 = Model $2+$ impaired cerebrovascular reactivity vs normal, carotid intima-media thickness, global cognitive score, and geriatric depression score $\geq 5$ at time 1 (2004-2009).

e Model 4 = Model 3 applying inverse probability weights.

${ }^{f}$ Model 5 = Model 3 after excluding 53 participants with stroke and dementia at time 2 (2011-2013).

The opinions expressed by authors contributing to this journal do not necessarily reflect the opinions of the U.S. Department of Health and Human Services, the Public Health Service, the Centers for Disease Control and Prevention, or the authors' affiliated institutions. 
Table 3. Multinomial Logistic Regression for Association Between BMI (as a Continuous Variable) and Sarcopenia Status Among a Sample of Men ( $\mathrm{n}=337$ ) Participating in Bezafibrate Infarction Prevention Neurocognitive Study, Israel, 2011-2013 ${ }^{\mathrm{a}}$

\begin{tabular}{|c|c|c|c|c|}
\hline \multirow[b]{2}{*}{ Model } & \multicolumn{2}{|c|}{ Probable Sarcopenia } & \multicolumn{2}{|c|}{ Sarcopenia } \\
\hline & OR (95\% Cl) & $P$ Value & OR $(95 \% \mathrm{Cl})$ & $P$ Value \\
\hline Model $1^{\mathrm{b}}$ & $1.24(1.11-1.39)$ & $<.001$ & $1.34(1.19-1.51)$ & $<.001$ \\
\hline Model $2^{C}$ & $1.28(1.12-1.45)$ & $<.001$ & $1.39(1.21-1.59)$ & $<.001$ \\
\hline Model $3^{d}$ & 1.33 (1.16-1.53) & $<.001$ & $1.38(1.19-1.59)$ & $<.001$ \\
\hline Model $4^{e}$ & $1.28(1.18-1.39)$ & $<.001$ & $1.34(1.23-1.45)$ & $<.001$ \\
\hline Model $5^{f}$ & $1.33(1.15-1.54)$ & $<.001$ & $1.42(1.21-1.66)$ & $<.001$ \\
\hline
\end{tabular}

Abbreviations: BMI, body mass index; OR, odds ratio.

${ }^{a}$ In all comparisons, reference outcome value is robust, defined as no evidence of sarcopenia.

${ }^{\mathrm{b}}$ Model 1 = age, education ( $\geq 12 \mathrm{y}$ vs $<12 \mathrm{y}$ ), birthplace (Europe, Middle East vs Israel).

${ }^{c}$ Model 2 = Model $1+$ systolic blood pressure (continuous), physical activity, diabetes, insulin resistance (top quartile vs others), C-reactive protein, high-density lipoprotein cholesterol, and triglycerides (continuous).

${ }^{d}$ Model $3=$ Model $2+$ impaired cerebrovascular reactivity vs normal, carotid intima-media thickness, global cognitive score, and geriatric despression score $\geq 5$ at time 1 (2004-2009).

${ }^{\mathrm{e}}$ Model 4 = Model 3 applying inverse probability weights.

${ }^{\mathrm{f}}$ Model 5 = Model 3 after excluding 53 participants with stroke and dementia at time 2 (2011-2013).

The opinions expressed by authors contributing to this journal do not necessarily reflect the opinions of the U.S. Department of Health and Human Services, the Public Health Service, the Centers for Disease Control and Prevention, or the authors' affiliated institutions. 